\title{
Management of interleukin-2-induced severe bronchoconstriction
}

\section{To the Editors:}

The immunostimulatory cytokine, interleukin (IL)-2, is used for the treatment of patients with cancers, such as metastatic renal cell carcinoma and melanoma $[1,2]$. The toxicity of IL-2 has been well described, including hypotension, a capillary leak syndrome including pulmonary oedema, thrombocytopenia and renal dysfunction [1,3-5]. Interestingly in a rare case, IL-2 administration, especially its inhalation, was reported to cause bronchoconstriction [3, 4, 6]. However, little is known about the risk factors and management of IL-2-induced bronchoconstriction. We conducted a study to elucidate the clinical characteristics of patients developing IL-2-induced bronchoconstriction and to examine how to manage this condition.

The study subjects included 18 patients with malignant cutaneous haemangioendothelioma (10 males and 8 females). Most of them were elderly with a mean (range) age of 72.3 (5295) yrs, and all had lesions on the face and head. All patients received IL-2 immunotherapy plus radiotherapy. IL-2 was administered intralesionally in four patients and intravenously in one. The remaining 13 patients received IL-2 both intralesionally and intravenously. IL-2 was usually given $2-3$ times a week. Total doses of intralesional IL-2 were $1.2-56 \times 10^{6}$ $\mathrm{U}$ (average, $13.8 \times 10^{6} \mathrm{U}$ ) and intravenous IL-2 were $8-60 \times 10^{6}$ $\mathrm{U}$ (average, $32.8 \times 10^{6} \mathrm{U}$ ).

During therapy, three patients developed severe cough, dyspnoea and chest tightness. The clinical characteristics of these three patients are shown in table 1. Intralesional administration of IL-2 was performed in two of these patients, while the third patient was given IL-2 intravenously. Chest auscultation showed a diffuse wheeze. Pulmonary function tests revealed obstructive impairment with no abnormal opacities on chest radiograph. Inhalation of the bronchodilator salbutamol, a short-acting $\beta_{2}$-agonist (SABA), showed a small increase in forced expiratory volume in one second (FEV1 $<10 \%$ ) with little symptomatic improvement (table 1). All patients developing bronchoconstriction had a history of asthma, but they were mild-intermittent asthmatics not needing regular medication, such as inhaled corticosteroids (ICS). Bronchoconstriction typically occurred 6-8 h after IL-2 administration, and continued overnight, regardless of the administration route (fig. 1). Inhalation of a SABA before IL-2 administration did not prevent the bronchoconstriction. Pretreatment with ICS (fluticasone propionate 200-400 $\mu \mathrm{g}$ b.i.d) alone or a long-acting $\beta_{2}$-agonist (LABA; salmeterol $50 \mu \mathrm{g}$ b.i.d) alone, which were given 1 day before IL-2 administration, decreased an IL-2-induced fall in FEV1 by $<30 \%$. However, pre-treatment with ICS plus a LABA (fluticasone propionate 200-400 $\mu \mathrm{g}$, salmeterol $50 \mu \mathrm{g}$ b.i.d) markedly reduced the decline in FEV1 by $70-80 \%$ with symptomatic improvement (fig. 1). The serum levels of IL-4, IL-5, eosinophilic cationic protein or the plasma levels of thromboxane- $B_{2}$ did not increase after IL-2 administration.

Bronchoconstriction has been reported as an adverse effect of IL-2 in several studies, but in the majority, IL-2 was administered by inhalation [6]. Our study demonstrated that the intravenous and even intralesional administration of IL-2 caused bronchoconstriction. Notably, IL-2-induced bronchoconstriction occurred exclusively in patients with a history of bronchial asthma, suggesting that asthmatics are more susceptible to bronchoconstriction induced by IL-2 therapy. IL-2-induced bronchoconstriction usually appeared 6-8 $\mathrm{h}$ after IL-2 administration with a $40-55 \%$ decrease of baseline FEV1 that lasted overnight. To date, there has been only one case report studying the treatment of IL-2-induced bronchoconstriction [6], in which pre-treatment with a SABA successfully prevented bronchoconstriction induced by inhaled IL-2 therapy in a case of metastatic lung tumours of renal cell carcinoma. However, the present study showed that SABA

TABLE 1 Characteristics of patients developing bronchoconstriction by interleukin (IL)-2 therapy

\begin{tabular}{|c|c|c|c|c|c|c|c|c|c|}
\hline \multirow[t]{2}{*}{ Patient } & \multirow[t]{2}{*}{ Sex } & \multirow[t]{2}{*}{ Age yrs } & \multirow{2}{*}{$\begin{array}{c}\text { History of } \\
\text { asthma }\end{array}$} & \multirow{2}{*}{$\begin{array}{c}\text { IL-2 } \\
\text { administration }\end{array}$} & \multirow[t]{2}{*}{$\operatorname{lgE~U} \cdot \mathrm{mL}^{-1}$} & \multirow[t]{2}{*}{$\mathrm{PC}_{20} \mathrm{mg} \cdot \mathrm{mL}^{-1}$} & \multicolumn{3}{|c|}{ FEV $_{1} \mathrm{~L}$} \\
\hline & & & & & & & Baseline & $\begin{array}{c}\text { After } \\
\text { IL-2 therapy }\end{array}$ & $\begin{array}{l}\text { Post-inhalation } \\
\text { of bronchodilato }\end{array}$ \\
\hline 1 & M & 69 & Yes & Intralesional + i.v. & 104 & 2.1 & 2.12 & 0.91 & 1.00 \\
\hline 2 & $\mathbf{F}$ & 67 & Yes & Intralesional & 86 & ND & 1.88 & 0.98 & 1.02 \\
\hline 3 & M & 59 & Yes & Intralesional + i.v. & 44 & 2.6 & 2.54 & 1.41 & 1.50 \\
\hline
\end{tabular}

Ig: immunoglobulin; PC20: methacholine concentration causing a 20\% fall in forced expiratory volume in one second (FEV1); M: male; F: female; ND: not done. 


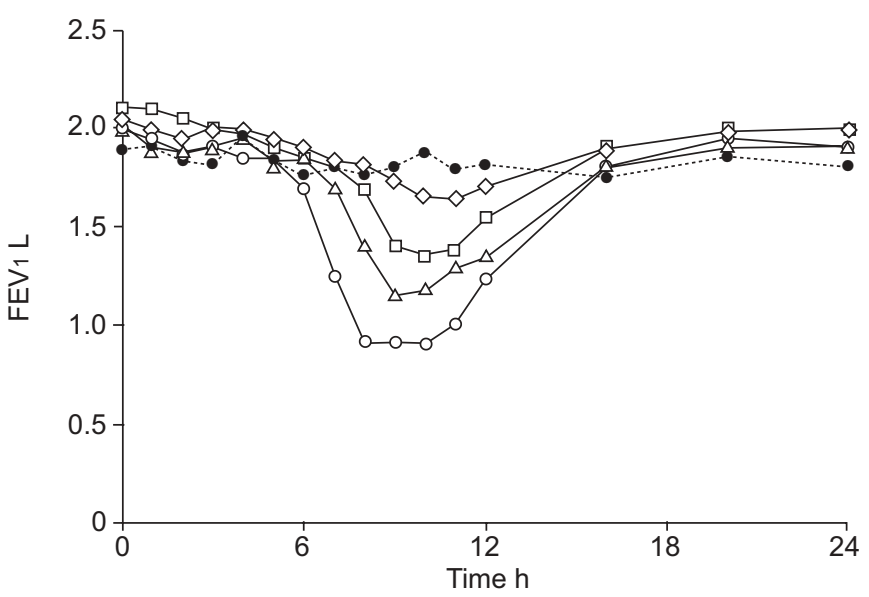

FIGURE 1. Changes in forced expiratory volume in one second (FEV1) during interleukin (IL)-2 therapy. Vehicle $(\bullet)$ or IL-2 $(\bigcirc)$ were injected intravenously into patient 1. Changes in FEV1 after pre-treatment with inhaled corticosteroid $(\triangle)$ or a long-acting $\beta_{2}$-agonist $(\square)$ alone, or in combination $(\diamond)$ are shown.

pre-medication failed to prevent the bronchoconstriction. The different route of IL-2 administration may partly explain this discrepancy. Inhalation of IL-2 was reported to develop bronchoconstriction very shortly after administration [6], while bronchoconstriction induced by intralesional and intravenous injection started 6-8 h after therapy and continued overnight. Thus, a SABA is unlikely to prevent the latter bronchoconstriction because of its short-acting effect. Interestingly, pretreatment with LABA or ICS alone induced only a small increase in an IL-2-reduced FEV1, but ICS in combination with a LABA markedly decreased a fall in FEV1 with symptomatic improvement. These results suggest that, in addition to a longacting bronchodilator, anti-inflammatory drugs were required for preventing IL-2-induced bronchoconstriction.

In summary, our study showed that interleukin-2 administration, even intralesionally, induced severe bronchoconstriction in patients with malignant cutaneous haemangioendothelioma. Bronchoconstriction occurred exclusively in patients with a history of bronchial asthma; therefore, attention must be directed to interleukin-2-induced bronchoconstriction, especially in patients with a history of asthma. Once it occurred, interleukin-2-induced bronchoconstriction did not respond well to bronchodilators, and pre-treatment with inhaled corticosteroids in combination with a long-acting $\beta_{2^{-}}$ agonist successfully prevented this condition.

T. Suda*, H. Hashizume", Y. Aoshima\#, K. Yokomura*, J. Sato*, N. Inui*, Y. Nakamura*, T. Fujisawa*, N. Enomoto* and K. Chida*

*Second Division, Dept of Internal Medicine, and "Dept of Dermatology, Hamamatsu University School of Medicine, Shizuoka, Japan.

\section{STATEMENT OF INTEREST}

None declared.

\section{REFERENCES}

1 Tarhini AA, Agarwala SS. Interleukin-2 for the treatment of melanoma. Curr Opin Investig Drugs 2005; 6: 1234-1239.

2 McDermott DF, Atkins MB. Interleukin-2 therapy of metastatic renal cell carcinoma: predictors of response. Semin Oncol 2006; 33: 583-587.

3 Huland E, Heinzer H, Huland H. Inhaled interleukin-2 in combination with low-dose systemic interleukin-2 and interferon alpha in patients with pulmonary metastatic renal-cell carcinoma: effectiveness and toxicity of mainly local treatment. J Cancer Res Clin Oncol 1994; 120: 221-228.

4 Gordon MS, Battiato LA, Gonin R, Harrison-Mann BC, Loehrer PJ Sr. A phase II trial of subcutaneously administered recombinant human interleukin-2 in patients with relapsed/refractory thymoma. J Immunother Emphasis Tumor Immunol 1995; 18: 179-184.

5 Guirguis LM, Yang JC, White DE, et al. Safety and efficacy of high-dose interleukin-2 therapy in patients with brain metastases. J Immunother 2002; 25: 82-87.

6 Barutca S, Meydan N, Barlak A. Prevention of interleukin-2induced severe bronchospasm with salbutamol. J Aerosol Med 2003; 16: 183-184. 